\title{
Interface Characterization of Different Types of Fibers in Engineered Cementitious Composites (ECC)
}

\author{
(A Review of the Basic Micromechanical Parameters of ECC)
}

\author{
J. J. Makwana ${ }^{1}$, Dr. J. D. Rathod ${ }^{2}$ \\ ${ }^{I}$ Civil Engineering Department, Rai University, Ahmedabad, Gujarat, INDIA \\ ${ }^{2}$ Applied Mechanics Department, M. S. University of Baroda, Vadodara, Gujarat, INDIA
}

\begin{abstract}
This paper surveys the research and development over the last decade about various active researches that are taking place around the world on study of interface characterization of different types of fibers used in ECC. Engineered cementitious composites (ECC), a unique member of HPFRCC featuring high tensile ductility. While micromechanics is applied in many aspects of the material design process, emphasis of this study is placed on the effect of fly ash content on altering material micro- structure and mechanical properties. Observations of micromechanical properties of interface characterization of ECC based on a large range of theoretical and experimental research are reviewed. Most of the researchers applied a single fiber pull-out test in different studies to measure the bond properties of fibers with different length, inclination and chemical treatment matrices. Outcomes of different researches carried out so far suggest that interface properties can be measured by Single fiber pull-out test. Further, a micromechanics model is developed to characterize the interface properties at single fiber pullout level. In the model, frictional bond strength, chemical debonding energy, slip-hardening and slip-softening coefficients are explicitly accounted for.
\end{abstract}

Keywords - Engineered Cementitious Composites, Micromechanics, Single fiber pull-out test, fibers, Minerals.

\section{Introduction}

Concrete is the most popular construction material. It is difficult to point out another material of construction which is as versatile as concrete. It is the material of choice where strength, permanence, durability, impermeability, fire resistance and abrasion resistance exists. The application of a very ductile fiber reinforced cementitious composite as the overlay material may overcome the commonly observed overlay durability problems. A series of experimental, analytical and numerical investigations carried out over the last several years point to a high potential of a much more durable repaired system when Engineered Cementitious Composites (ECC) are used in place of normal concrete. After a brief introduction to ECC, this project first reviews research on the interface property characterization. In the last several decades, concrete with increasingly high compressive strength have been used for structural applications. However, most of these materials remain brittle. In some cases, the brittleness as measured by the brittleness number actually increases as the compressive strength goes up. In the last several years, many researchers have been investigating a composite material known as Engineered Cementitious Composites, or ECC in short. It is a fiber reinforced cement based composite material systematically engineered to achieve high ductility under tensile, flexure and shear loading. ECC is a special type of high performance fiber reinforced cementitious composites (HPFRCC) featuring significant tensile ductility.

The design of ECC is guided by micromechanics models, which provide quantitative links between composite mechanical behavior and the properties of the individual phases, i.e., fiber, matrix and interface. Utilizing the models, the desired high tensile ductility, which is achieved through strain-hardening and multiple cracking, is converted to a set of constraints on individual component properties. These components, i.e. the fiber, the matrix and the interface, are then synergistically tailored to meet the constraints. The high ductility of ECC is achieved with minimum fiber volume fraction by a structure tailoring of matrix, interface and fiber properties. Interface is one of the most important mechanical parameter which affects composite behavior and governed by effective bond between fiber and matrix.

ECC is a fiber reinforced cement based composite material systematically engineered to achieve high ductility under tensile, flexure and shear loading. By employing micromechanics-based material design, maximum ductility in excess of $3 \%$ under different loading can be attained with $2 \%$ fiber content by volume. This moderate amount of short discontinuous fibers allows flexibility in construction execution, including selfconsolidation casting and shotcreting. The characteristic strain hardening after first cracking is accompanied by multiple micro cracking.

In this survey, it is a generic micromechanics model that can be used for almost all the fiber types, i.e. nature of fibers with hydrophilic and hydrophobic. The fiber bridging constitutive law $\sigma(\delta)$ is employed in both strain hardening criteria. It determines the complementary energy $\mathrm{J}_{\mathrm{b}}{ }_{\mathrm{b}}$ as well as the maximum bridging strength 
$\sigma_{0}$. Therefore, the $\sigma(\delta)$ relationship critically controls the tensile strain-hardening behavior of ECC. Fiber bridging constitutive law $\sigma(\delta)$ describes the relationship between the bridging stress $\sigma$ transferred across a crack and the opening of this crack $\delta$. In the development of ECC, the $\sigma(\delta)$ relationship is of primary importance. Therefore, control of $\sigma(\delta)$ curve through tailoring material microstructure is the key to successfully design ECC material. The composite beahviour of ECC is governed by the total contribution of the single fiber interaction with the matrix. If mechanics involved in the composite behavior could be described theoretically it would be possible to predict the bulk behavior of the matrix from the single fiber pull-out performance. Such an approach is beneficial as it enables optimization of the combination of fiber type and cementitious matrix. It is generally considered in literature that there exists no ideal system of testing and modeling in order to fully predict mechanical behavior of composites even for the basic case of uniaxial tension. However, the results from pullout tests may always be used for a qualitative comparison between fiber types, fiber dimensions and concrete mixes. Further, activities in finding a way to map the single fiber behaviour to the bulk behaviour may lead to a basic understanding of the mechanisms involved.

Fiber pull-out tests are often used to study the fiber-matrix interaction behavior in Fiber Reinforced Cement Composites (FRC), a factor strongly influencing the properties of the composites. The pull-out test is also important by itself as it simulates the fiber bridging-pull-out phenomenon during the fracture process of FRC. Fiber pull-out is often modeled with elastic bond strength and frictional bond strength for the fiber-matrix interface. However, the frictional bond strength generally varies with the fiber slippage distance relative to the matrix. Fiber-matrix interface properties are experimentally determined through a load-displacement relation of a fiber pull-out of a matrix. Fiber debonding and pull-out at the interface have a significant influence on total energy absorption during crack propagation.

Interface properties can be measured by Single fiber pull-out test. A single fiber pull-out test is carried out in this study to measure the bond properties of fibers with different length, inclination and chemical treatment matrices. Proper characterization of the interface properties via micromechanics models can be lead to effective tools for designing high performance and cost-effective cement based materials.

\section{Single Fiber Pull-Out Test}

A method to directly assess fiber/mortar interfacial bond properties is to pull a single fiber out of its surrounding matrix. The bond properties are mainly described through the notions of chemical debonding energy $G_{d}$ of frictional bond strength at the onset of fiber slippage, $\tau_{0}$, and slip-hardening or slip-softening coefficient, $\beta$. Fiber-matrix interfacial properties are important in controlling macroscopic properties of composite materials. Generally speaking, better the bond better is the composite. Increasing the bond leads to increase in both composite strength and toughness $[1,2]$. However, beyond certain bond strength, toughness begins to decrease due to fiber rupture. Therefore, fiber pulls out rather than rupture confers a larger ductility to the fiber reinforced composite [3-5]. In addition, the phenomenon of pseudo strain hardening is strongly influenced by the maximum bridging stress, which is directly proportional to the interface bond strength $[6,7]$.

A commonly used technique to investigate fiber-matrix interfacial behaviors is single fiber pullout. Fig. 1 shows schematically the set-up for a typical single fiber pull out test. The specimens are fabricated according to the technique for microfibers described in [8].

The general profile of a single fiber pull out curve can be decomposed into three major regimes as depicted in Fig. 2 [9]. Initially a stable fiber debonding process occurs along the fiber/matrix interface. The load resisted by the fiber is then increasing up to $\mathrm{P}_{\mathrm{a}}$. The fiber embedded end, $\mathrm{l}=\mathrm{l}_{\mathrm{e}}$, does not move. The debond length, $l_{\mathrm{d}}$, increase towards $l_{\mathrm{d}}=l_{\mathrm{e}}$. The displacement corresponds only to elastic stretching of the debonded fiber segment and of the fiber free length. Then, the load decreases form $\mathrm{P}_{\mathrm{a}}$ to $\mathrm{P}_{\mathrm{b}}$.

If the load drop is significant, it reveals that the chemical bond between the fiber and the matrix has broken. This means that debonding at the interface is governed by a fracture criterion [10]. In the case of nonchemically bonded fibers, such as steel, polyester, or polypropylene fibers, $\mathrm{P}_{\mathrm{a}}$ would be closed or equal to $P_{b}$. At point $P_{b}$, the embedded fiber end is just debonded. Finally, in the slippage regime, the fiber load is resisted by frictional forces. The fiber can undergo sliding with either slip hardening, constant friction or slip softening effect, characterized by the coefficient $\beta$, which is, respectively, positive, zero, or negative [3]. Slip hardening may occur with polymer fibers, if they are less hard than surrounding matrix. They would get damaged and a jamming effect can take place inside the matrix. This leads to an increasing load resisting fiber pull out. This phenomenon can be very beneficial as long as fiber tensile strength is not exceeded. Conversely, constant friction or slip softening is often observed when the fiber hardness is higher than that of the surrounding matrix. Frictional bond strength $(\tau)$ can be computed as $\mathrm{P}_{\mathrm{b}} / \pi \mathrm{d}_{\mathrm{f}} \mathrm{l}_{\mathrm{e}}$ when full debonding of fiber takes place with a constant friction. 


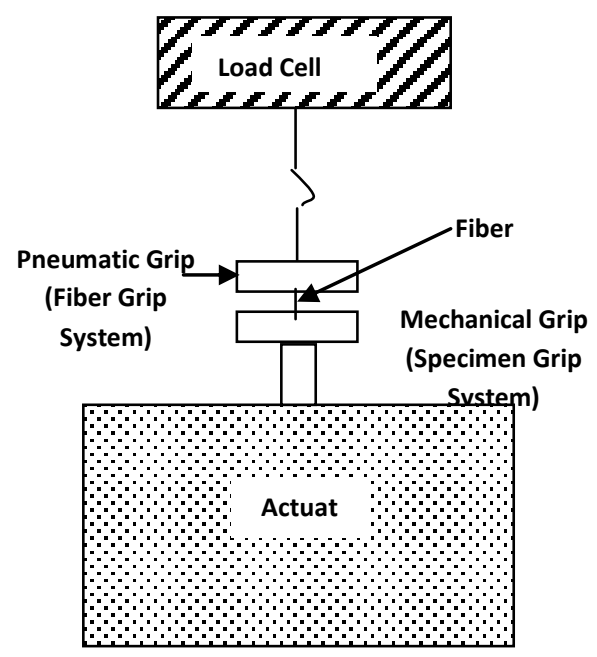

Fig. 1. Schematically the Test set-up for a typical single fiber pull-out test

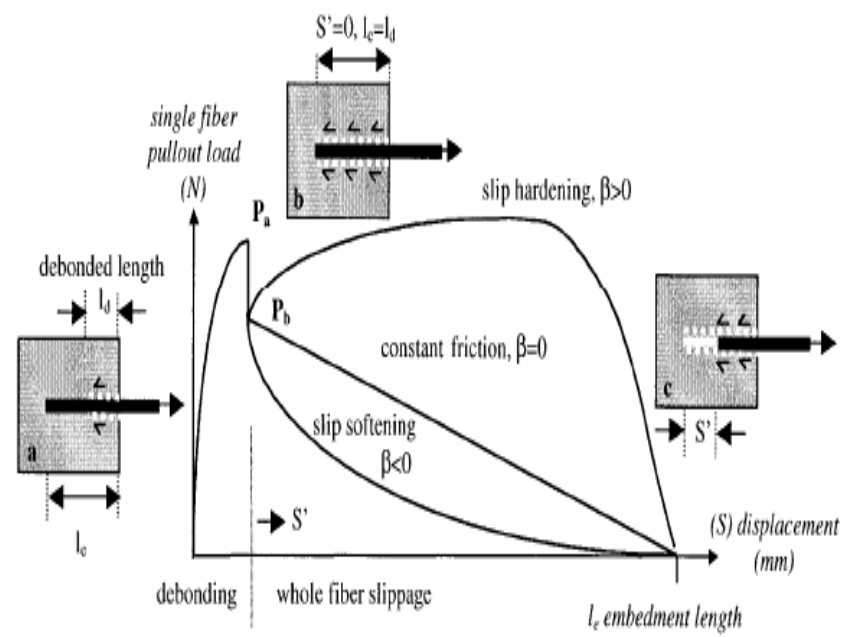

Fig. 2. General Profile of Single Pullout Curve

\section{Objectives}

- To review the ingredients of Engineered Cementitious Composites (ECC).

- To study the micromechanical interface properties of Engineered Cementitious Composites (ECC).

\section{Ingredients of ECC}

The mixture design of ECC is the key factor, which to a large extent determines its performance on the structural level. Also, a standard mixture design for high performance concrete does not exist - for each specific application a different mixture design is required. Generally, course aggregates are not used in the mix design. ECC mix design involves the ordinary Portland cement, Fine aggregates, different types fibers, Water, Super plasticizer and Mineral admixtures (Fly Ash).

\section{Survey on Interface Characterization of ECC}

In the last several years, many researchers have been investigating a composite material known as Engineered Cementitious Composites, or ECC in short. It is a fiber reinforced cement based composite material systematically engineered to achieve high ductility under tensile, flexure and shear loading. ECC is a special type of high performance fiber reinforced cementitious composites (HPFRCC) featuring significant tensile ductility.

The design of ECC is guided by micromechanics models, which provide quantitative links between composite mechanical behavior and the properties of the individual phases, i.e., fiber, matrix and interface. Utilizing the models, the desired high tensile ductility, which is achieved through strain-hardening and multiple cracking, is converted to a set of constraints on individual component properties. These components, i.e. the fiber, the matrix and the interface, are then synergistically tailored to meet the constraints. The high ductility of ECC is achieved with minimum fiber volume fraction by a structure tailoring of matrix, interface and fiber properties. Interface is one of the most important mechanical parameter which affects composite behavior and governed by effective bond between fiber and matrix.

Victor C. Li [11] reviewed the development of pseudo strain hardening cement based short fiber composites employing the Performance Driven Design Approach. The micromechanics theory behind the design concept was reviewed, and the unique mechanical properties of the resulting composite were summarized. The translation of material properties to structural properties was demonstrated with the structural response of the Ohno Shear beam. He surveyed the adoption of the PDDA approach in the development of a strain hardening ECC designed for shear intensive structures. Micromechanics tools provide a major link between structural performance and material structure tailoring. By proper selection of fiber, matrix and interface, it was demonstrated that a composite with improved strength, strain and fracture energy properties can be manufactured and that the amount of fiber reinforcement did not necessarily have to be excessively high. This last aspect makes it cost effective. ECCs with steel, carbon and other polymeric fiber types were investigated.

A brief overview of several classes of fiber reinforced cement based composites and suggested future direction in FRC development. Special focus was placed on micromechanics based design methodology of strain-hardening cement based composites. As example, a particular engineered cementitious composite newly 
developed at the ACE-MRL at the University of Michigan was described in detail with regard to its design, material composition, processing, and mechanical properties. Three potential applications which utilize the unique properties of such composites were cited in the paper, and future research needs were identified by Victor C. Li [12].

In 2003, Victor C. Li [13] surveyed in their article the research and development of Engineered Cementitious Composites (ECC) over the last decade since its invention in the early 1990's. The importance of micromechanics in the material design strategy is emphasized. The advantageous of ECC based on a broad range of theoretical and experimental research are examined. The advantageous use of ECC in certain categories of structural, and repair and retrofit applications is reviewed. While reflecting on past advances, future challenges for continued development and deployment of ECC are noted. This article is based on a keynote address given at International Workshop on Ductile Reinforced Cementitious Composites (DFRCC).

The theoretical analyses of fiber pullout from a matrix reported. The effects of Poisson's ratio, elastic frictional bond strengths and bond strength variation with slippage distance on the pullout relation were also reviewed. The model takes into consideration the variation of the frictional fiber matrix bond strength with fiber slippage distance. For the synthetic fibers, the bond strength was found to increase with the slippage distance during the process of pullout reviewed by the Wang, Li and Backer [14].

Victor C. Li, Wang and Backer [15] in an experimental investigation of synthetic fiber pull-out at an angle observed that the force and energy of fiber pull-out increase with the inclination angle, but such increases are limited by the strength of the fiber cement matrix at high angles due to matrix spalling. Studies were also conducted on the effects of fiber bundling and surface treatment on the pull-out behaviour. It is suggested that for effective use of the reinforcing fibers, fiber bundling should be minimized and the fiber/matrix bond property should be controlled. They concluded that the effect of fiber pullout at an angle on the pullout load can be reasonably represented by a snubbing friction coefficient.

In 1995, Li, Maalej, and Hashida [16] presented a probabilistic-based micromechanical model developed for the post cracking behaviour of a brittle matrix reinforced with short, randomly distributed fibers. The model that predicts the composite bridging stress crack-opening (COD) relationship, accounts for fiber pullout, fiber tensile rapture and a local frictional effect called snubbing. However, it does not account for fiber bending rapture, and the possible effect of matrix spalling at the exit points of inclined fibers from the matrix. The model assumes a fiber/matrix interface that is controlled by a constant frictional bond stress. The model is used to predict the composite tensile strength and fracture energy. Comparisons of model-predicated bridging stress-COD relationship with experimental data, where fiber rapture has occurred, show reasonable agreement supporting the validity of the proposed model. The model is then used to perform a parametric study to evaluate the effect of the micromechanical parameters on the composite tensile strength and fracture energy. The study suggests that this model can be used to design the composite for optimum performance.

Amnon Katz, Victor C. Li, and A. Kazmer [17] tested the interfacial bond properties of two carbon fibers, having diameters of $10 \mu \mathrm{m}$ and $46 \mu \mathrm{m}$, for cement matrices of different water-to-binder $(\mathrm{w} / \mathrm{b})$ ratios and silica fume contents. The pull-out test was conducted using a special technique that prevented the brittle fiber from breaking during specimen handling. An environmental scanning electron microscope (ESEM) was used to determine the nature of the fiber-matrix bond. The results show a friction-based bond mechanism for the fiber of $10 \mu \mathrm{m}$, with bond strength of $0.5 \mathrm{MPa}$ for the high w/b matrix and without silica fume. Densifying the matrix by lowering the $\mathrm{w} / \mathrm{b}$ ratio or by using silica fume improved the bond by $50-100 \%$. For the large-diameter fiber, silica fume had a very strong effect on the bond (an increase of 370-670\%). It appears that long grooves along the fiber surface crates a mechanical anchorage, which is strongly affected by the presence of silica fume.

In this research paper, they have derived relationship between the pull-out load and the displacement of a fiber when its pulls out of the matrix serves as an important parameter in the design of composite materials. Many analytical models for the predication of the behaviour of composite materials are based on the assumption of a constant friction load between the fiber and the matrix, regardless of the displacement of the fiber. Based on this assumption test techniques were developed in which the critical length of the fiber was determined. The bond strength was calculated from the critical length assuming that the fiber strength and diameter are known. In this work, they have used carbon fiber A. Katz and Victor C. Li [8].

In 1997, Chan and Li [18] reported findings of an investigation on the age development of interfacial properties of polyethylene fibers in a cementitious matrix. It was found that the interfacial bond strength matures much faster, in less than 7 days, in comparison with bulk property development, which typically takes 14-28 days. A parallel SEM study of the microstructure development in the interfacial transition zone supported the fact that the rapid early age saturation of the interfacial adhesive bond strength is associated with the growth of a $\mathrm{CH}$ rim around the fiber periphery. The formation of the $\mathrm{CH}$ layer appears to be completed much earlier than the hydration process of the bulk material. The slip-dependent friction after initial debonding was found to develop with age up to 28 days. Gradually converting from a slip-weakening to a slip-hardening behaviour. These findings should be useful in interpreting early age the fiber-reinforced cementitious composite properties. 
Kanda and Li [19] presented the characterization of fiber/matrix interfacial properties and the apparent strength of high-strength hydrophilic fibers. Single fiber pull-out bond tests and single fiber pull-torupture strength tests were conducted by employing PVA fibers. The pull-out bond tests showed that these fibers have surprisingly high chemical and frictional bond strengths. The chemical bond strength was relatively stable independent of a water-to-cement ratio of matrix and the fiber type tested, contrary to the frictional bond strength. The pull-to-rupture strength tests revealed that the apparent strength of the PVA fibers in cementitious composites is considerably lower than that in standard fiber strength tests. The apparent strength was further reduced with inclining angle of fiber alignment. This effect was captured by a simple phenomenon logical model which introduces the apparent strength reduction factor. The combined effects of high bond strength and degraded fiber strength will likely contribute to performance less than would be expected from a highperformance fiber.

In 2001, Redon, Li, Wu, Hoshiro, Saito and Ogawa [20] used a single fiber pullout test to measure the bond properties of polyvinyl alcohol fibers that are available at various diameters in a mortar matrix. Despite short fiber embedment lengths, the small diameter fibers ruptured during the pullout tests. However, it is shown that even if full fiber pullout is not achieved, it is still possible to determine a chemical debonding energy, $G_{d}$, and initial interfacial frictional bond strength, $\tau_{0}$. Despite high $\mathrm{G}_{\mathrm{d}}$ values, the fibers did not rupture during the fiber chemical debonding process, but during fiber pull-out, a strong slip-hardening effect, characterized by the high values of the slip-hardening coefficient, $\beta$, and induced severe abrasion damage visible under scanning electron microscope on the fiber surface. As a consequence, when the fiber apparent tensile strength was exceeded, fibers ruptured by delamination. Finally, an attempt was made to lower the values of the bond properties to minimize fiber rupture during pullout. This goal was partially achieved by applying an oil coating on the hydrophilic polyvinyl alcohol fiber surface to reduce any strong fiber/mortar interaction.

He gave a brief summary of the main properties and limitations of steel fibers used in cement based composites and also described the rational and technical background behind the development and design of a new generation of steel fibers for use in cement, ceramic and polymeric matrices. These fibers were engineered to achieve optimal properties in terms of shape, size, and mechanical properties, as well as compatibility with a given matrix. They are identified as Torex fibers. Typical tests results are provided and illustrate without any doubt the superior performance ( 2 to 3 times) of Torex fibers in comparison to other steel fibers on the market. Reported by Antoine E. Naaman [21].

Victor C. Li, Tetsuo Horikoshi, Atsuhisa Ogawa, Shinichi Torigoe, and Tadashi Saito [22], in their research paper mentioned that the durability of engineered cementitious composites (ECC) reinforced with polyvinyl alcohol (PVA) fiber is investigated in this paper. ECCs have been realized as ductile strain-hardening cementitious composites with tensile strain capacity up to 5\%. This material is being applied in new construction and for the repair and retrofit of structures. A micromechanics-based approach is adopted in the present durability study. The micromechanics-based model relates the fiber, matrix, and interface parameters to composite properties through knowledge of microdeformation mechanisms beyond the elastic stage. Composite property changes resulting from environmental loading are expected to be a manifestation of changes in properties at the fiber, matrix, and/ or interface level. This concept is examined in this paper by experimentally determining the changes in the fiber and fiber- matrix interface properties with specimens exposed to accelerated testing and correlating such changes to changes in the ductility of composites exposed to the same accelerated testing conditions. The accelerated test used in this study is a hot water immersion test simulating a long-term hot and humid environment. It is found that the fiber-matrix interface chemical bond increases while the apparent fiber strength decreases when the exposure time reaches 26 weeks. Correspondingly the composite ductility also decreases. The micromechanical model provides a rational means of interpreting and correlating the data from these two levels of testing. Despite the deterioration, PVA-ECC is found to retain tensile ductility more than 200 times that of normal concrete or normal fiber- reinforced concrete after exposure to an equivalent of 70 years or more of hot and humid environmental conditions.

This paper reports the microscale investigation of a new fiber-reinforced cementitious composite, highstrength, high-ductility concrete (HSHDC), which possesses a rare combination of very high compressive strength (166 MPa [24.1 ksi]) and very high tensile ductility (3.4\% strain capacity). The investigation involved experimental determination of fiber/matrix interaction properties using single-fiber pullout tests. A new mechanism of inclination-dependent hardening in fiber pullout - unique for a high-strength cementitious matrix - is discovered. The existing fiber-pullout analytical model for strain-hardening cementitious composites (SHCCs) is modified to incorporate the new mechanism. The modeled fiber-pullout behavior is used in a scalelinking model to compute the crack bridging $(\sigma-\delta)$ relation of HSHDC, which is also empirically verified through single-crack tests. The $\sigma-\delta$ relation of HSHDC satisfies the micromechanics-based necessary strength and energy conditions of steady-state flat crack propagation that prevent localized fracture. The microscale investigation of HSHDC in this research thus demonstrates the rational basis for its design combining both high 
compressive strength and high tensile ductility. Reported by Ravi Ranade, Victor C. Li, Michael D. Stults, Todd S. Rushing, Jason Roth, and William F. Heard (2013) [23].

In 2014, Xia Zhao, and Xiong - Jun He [24] reports the Flexural strength, impact resistance, and permeability and abrasion resistance performance of PVA fiber-reinforced concrete were measured as a function of fiber concentration. The proportions of PVA fibers in concrete matrix were $0,0.3,0.5,0.8,1.0,1.3$ and $1.5 \%$ volume fraction of concrete. The results showed that, with the introduction of PVA fibers, the flexural toughness and impact resistance of each prepared PVA fiber reinforced composites were improved. Compared to the reference sample, modulus of rupture, toughness indices, fracture energy, number of blows and impact energy of PVA fiber reinforced composites all were improved. The absorbed fracture energy and impact energy were provided by the mechanisms of matrix cracking, PVA fiber/matrix interface debonding, fiber pull-out and fiber rupture. The SEM analysis showed that the introduced PVA fiber acted as bridge across the crack, which improved the load-transfer efficiency. In addition, the permeability and abrasion resistance properties were also improved. However, deterioration was occurred in composites with 1.5 vol. \% PVA fibers. This reduction might be caused by the correspondingly introduced poor pore parameters and the loss of mix workability.

Srinivasa. C. H. and Dr. Venkatesh [25], presents facts about various research activities that are taking place around the world since the last one decade on development and study of behaviour of Engineered Cementitious Composites (ECC) using Polyvinyl Alcohol (PVA) fiber. Engineered Cementitious Composites can be designed based on micromechanical model with strain capacity of about 3 to $5 \%$ compared to $0.01 \%$ of normal concrete. The volume fraction of the fiber is less than 2 percent which shows extensive strain hardening behaviour of the composites.

Rathod JD [26], Study of effect of single fiber pull out test result on flexural performance of Engineered Cementitious Composite (ECC) is carried out. Different cement: sand ratios are used in composition of ECC. First crack strength, ultimate strength, toughness index, deflection hardening and reserved flexural strength are evaluated. Single fiber pull out test of $6 \mathrm{~mm}$ embedded length is carried out to evaluate frictional bond strength. Also he suggested an equation to evaluate ultimate flexural strength of ECC considering effect of micromechanical parameters in general and frictional bond strength in particular.

J. D. Rathod [27], in this innovative research and development paper reported that Pull out behaviour of steel fibers which are not straight and not circular in shape have received little attention compared to the straight steel fibers. Flat corrugated steel fibers are proved to be very effective at interface during pull out, which results into strain hardening and dissipation of significant amount of cold work energy. Such a performance encourages in development of a unique HPFRCC which is well known as Engineered Cementitious Composite (ECC). All such capabilities can be thoroughly explored by performing single fiber pull out test. In the present experimental study, a single fiber pull out test was performed on MTS machine under displacement control with specially designed fixtures to grip any type of fiber. Corrugated flat steel fibers were embedded in cementitious matrices with $6 \mathrm{~mm}$ and $12.5 \mathrm{~mm}$ lengths. Different cement and sand ratios were adopted along with replacement of cement by fly ash. Load was recorded at peak and corresponding pull out energy is calculated. De-bonding and pull out behaviour are evaluated qualitatively. Effects of fiber length, cement and sand ratio and fly ash percentage on pull out behaviour of flat corrugated fiber in cementitious composite are discussed in detail. This will help in developing the corrugated steel fiber based ECC.

\section{Conclusion}

- Micromechanical interface properties like chemical debonding energy, frictional bond strength, sliphardening and slip-softening coefficient can be determined from a single fiber pullout test.

- An analytical bridging stress-crack opening relation is derived, which can be used as a materials design tool for desired properties and cost-effectiveness via factors affecting the pullout beahviour of fiber selection (shape, diameter, embedded length, Aspect ratio, fiber bond factor, material, strength, pullout rate) matrix modification and fiber/matrix interface tailoring.

- Micromechanical study of strain hardening ECC provides useful guideline for microstructure tailoring. The effective guidance for interface property tailoring for high $\mathrm{J}_{\mathrm{b}}$ ' is revealed with the help of fly ash.

- High ductility of short composite requires large complementary energy and that becomes very important design criterion for ductile fiber composites. Using generic micromechanics principle, one can easily compare this quantity with different combinations of fiber, matrix, and interface properties and choose the one that satisfies required conditions to guarantee the ductility of such engineered fiber composites.

- The comparison between the two fiber/cement composites hydrophobic and hydrophilic is meant to demonstrate the usefulness of the model in fiber section for desired properties rather than just a comparison.

- Flexural Strength, impact resistance, permeability and abrasion resistance performance on PVA fiberreinforced concrete were measured as a function of fiber concentration. The absorbed fracture energy and impact energy were provided by the mechanics of matrix cracking, PVA fiber/matrix interface debonding, fiber pull-out and fiber rupture. Further, we may obtain more characterizations and details of 
micromechanical investigation on high strength, high-ductility concrete by replacing PVA fiber with different types of fibers.

\section{References}

[1] Bentur A., Mindess S. "Fiber reinforced cementitious composites", Elsevier Science Publishers, Amsterdam, Netherland, 1990.

[2] Li V. C., Wang Y, Backer S., "A micromechanical model of tension softening and bridging toughening of short random fiber reinforced brittle matrix composites", J. of Mech. and Physics of Solids 39: pp. 607-625, 1991.

[3] Cooper G. A., Kelly A., "The contribution to the work of fracture of a composite material of pull-out fibers", Mechanics of Composite Materials Wendt FW, Liebowitz H, Perrone L, eds., Pergamon, Oxford, U.K. pp. 653-661, 1970.

[4] Lin Z., Li V. C., "Crack bridging in fiber reinforced cementitious composites with slip-hardening interfaces", J. of Mech. Phys. Solids, Vol. 45(5), pp. 763-787, 1997.

[5] Li V. C., Kanda T., Lin Z., "The influence of fiber/matrix interface properties on complementary energy and composite damage tolerance", Proc., $3^{\text {rd }}$ Hong Kong. Conf. on Fracture and Strength of Solids, 1997.

[6] Li V. C., Leung CKY, "Steady-state and multiple cracking of short random fiber composite", J. of fiber composite,J. of Engg. Mech., ASCE 118, pp. 2246-2264, 1992.

[7] Li V. C., "From micromechanics to structural engineering-the design of cementitious composites for civil engineering applications", J. of Struct. Mech. and Earthquake Engg., 10, pp. 37-48, 1993.

[8] A. Katz, V. C. Li, " A Special Technique for determining the bond strength of micro-fibers in cement matrix by pullout test", J. of material science letters 15, pp. 1821-1823, 1996.

[9] Li V. C., Stang H., "Interface property characterization and strengthening mechanism in fiber reinforced cement based composites", J. of Advanced Cement Based Materials, Vol. 6(2), pp. 1-20, 1996.

[10] Leung CKY, Li V. C., "Strength-based and fracture based approaches in the analysis of fiber debonding", J. of Mat. Sci. Letter 9: pp. 1140-1142, 1990.

[11] Li V. C., "From micromechanics to structural engineering - The design of cementitious composites for civil engineering applications", Journal of Structural Mechanics and Earthquake Engg., Japan Society of Civil Engineers, Vol. 10, No. 2, pp. 37-48, 1993.

[12] Li V. C., ““Engineered cementitious composites (ECC) - tailored composites through micromechanical modeling”, Proceedings of Reinforced Concrete: Present and the Future, Eds: N. Banthia, A. Bentur, and A. Mufti, Canadian Society of Civil Engineers, 1997.

[13] Li V. C., "On engineered cementitious composites (ECC) - A review of the material and its applications", Journal of Advanced Concrete Technology, November, Vol. 1, No. 3, pp. 215-230, 2003.

[14] Wang Y., Li V. C. and Backer S., "Modelling of fiber pullout from a cement matrix", The international journal of cement composites and lightweight concrete, Volume 10 Number 3, pp. 143-149, 1988.

[15] Li V. C., Wang Y. and Backer S., "Effect of inclining angle, bundling and surface treatment on synthetic fiber pull-out from a cement matrix", Composites, Vol. 21, No. 2, pp. 132-140, March 1990.

[16] Li V. C., Maalej. M. and Hashida T., "Effect of fiber rupture on tensile properties of short fiber composites", Journal of Engineering Mechanics, pp. 903-913, 1995

[17] Li V. C., Katz A. and Kazmer A., "Bond properties of carbon fibers in cementitious matrix", Journal of Materials in Civil Engineering, Vol. 7, No. 2, pp. 125-128, May 1995.

[18] Li V. C. and Chan Y. W., "Age effect on the characteristics of fiber/cement interfacial properties", Advanced Civil Engineering Materials Research Laboratory, University of Michigan, pp. 5287-5292, 1997.

[19] Kanda T. and Li V. C., "Interface property and apparent strength of high-strength hydrophilic fiber in cement matrix", Journal of Materials in Civil Engineering, pp. 5-13, 1998.

[20] Carl Redon, Victor C. Li, Cynthia Wu, Hideki Hoshiro, Tadashi Saito, and Atsuhisa Ogawa, "Measuring and modifying interface properties of PVA fibers in ECC matrix", Journal of materials in civil engineering, pp. 399-406, 2001.

[21] Naaman A. E., "Engineered steel fibers with optimal properties for reinforcement of cement composites", Journal of Advanced Concrete Technology, Vol. 1, No. 3, pp 241-252, Nov. 2003.

[22] Victor C. Li, Tetsuo Horikoshi, Atsuhisa Ogawa, Shinichi Torigoe, and Tadashi Saito, "Micromechanics -based durability study of polyvinyl Alcohol-Engineered cementitious composites", ACI material journals, pp. 242-248, 2004.

[23] Ravi Ranade, Victor C. Li, Michael D. Stults, Todd S. Rushing, Jason Roth, and William F. Heard, "Micromechanics of Highstrength, High-Ductility concrete", ACI material journals, pp. 375-384, 2013.

[24] Xia Zhao and Xiong-Jun He, "High-toughness and durability performance characterization of concrete reinforced with poly(vinyl alcohol) fibers", Material Express, Vol. 4, No. 3, 2014.

[25] Srinivasa. C. H. and Dr. Venkatesh, "A Literature Review on Engineered Cementitious Composites for Structural Applications", International Journal of Engineering Research \& technology (IJERT), ISSN: 2278-0181, Vol. 3, Issue 12, December 2014.

[26] Rathod JD, "Effect of single fiber pull out test result on flexural performance of ECC", J. of civil \& environmental engineering, volume 4, Issue 2, 2014.

[27] J. D. Rathod, "Factors effecting pull out beahviour of corrugated steel fibers in cementitious composites", International journal of innovative research \& development, pp. 216-222, Volume 3, issue 2, 2014. 